LA W RENCE LIVERMORE N A TIO NAL LABORATORY

L. Kesler August 21, 2012

Laser wakefield acceleration self-guiding in noble gas mixes 
This document was prepared as an account of work sponsored by an agency of the United States government. Neither the United States government nor Lawrence Livermore National Security, LLC, nor any of their employees makes any warranty, expressed or implied, or assumes any legal liability or responsibility for the accuracy, completeness, or usefulness of any information, apparatus, product, or process disclosed, or represents that its use would not infringe privately owned rights. Reference herein to any specific commercial product, process, or service by trade name, trademark, manufacturer, or otherwise does not necessarily constitute or imply its endorsement, recommendation, or favoring by the United States government or Lawrence Livermore National Security, LLC. The views and opinions of authors expressed herein do not necessarily state or reflect those of the United States government or Lawrence Livermore National Security, LLC, and shall not be used for advertising or product endorsement purposes.

This work performed under the auspices of the U.S. Department of Energy by Lawrence Livermore National Laboratory under Contract DE-AC52-07NA27344. 


\title{
Laser wakefield acceleration self-guiding in noble gas mixes
}

\author{
Leigh A. Kesler \\ Office of Science, Science Undergraduate Laboratory Internship Program \\ Massachusetts Institute of Technology, Cambridge, Ma \\ Lawrence Livermore National Laboratory, Livermore, Ca
}

$8 / 10 / 2012$

Participant:

\section{Signature}

Supervisor:

\section{Signature}

Prepared in fulfillment of the requirement of the Office of Science, Department of Energy's Science Undergraduate Laboratory Internship under the direction of Dr. Siegfried Glenzer in the NIF Directorate at Lawrence Livermore National Laboratory.

This work performed under the auspices of the U.S. Department of Energy by Lawrence Livermore National Laboratory under Contract DE-AC52-07NA27344. 


\section{CONTENTS}

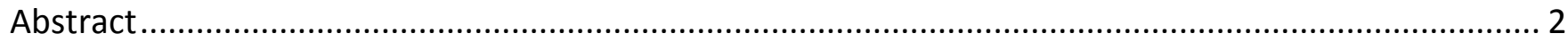

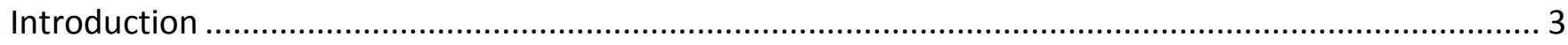

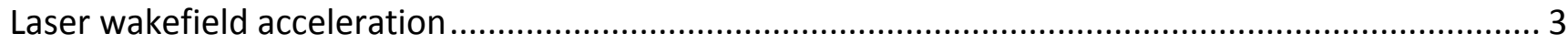

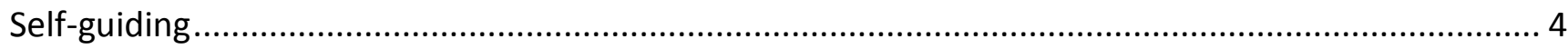

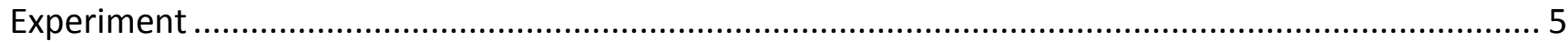

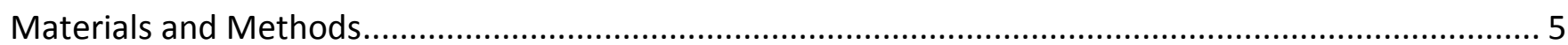

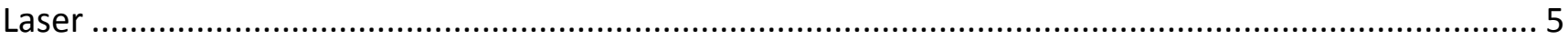

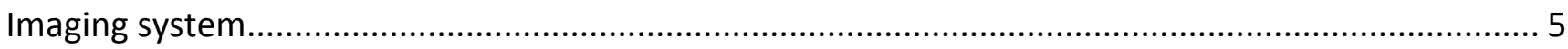

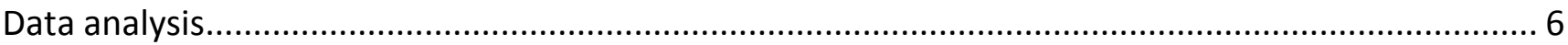

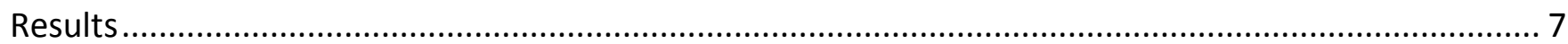

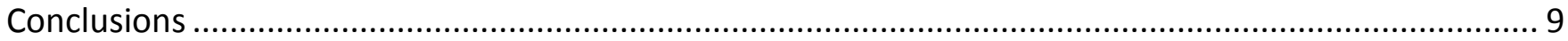

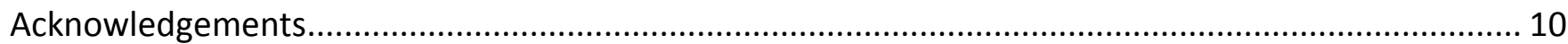

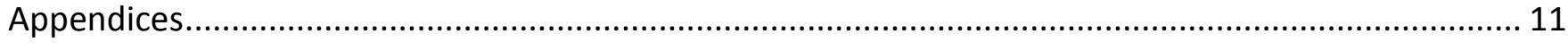

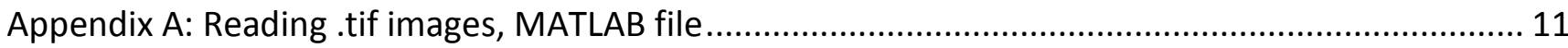

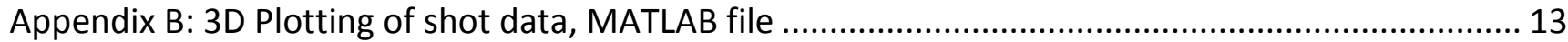

Appendix C: Maximum and FWHM calculation, MATLAB file ................................................ 13

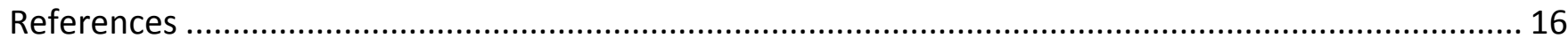




\begin{abstract}
Laser wakefield acceleration (LWFA) is a technique for accelerating charged particles to high energies over centimeter-scale distances, which has led to a new breed of compact particle accelerators. In order to keep laser intensity at $\sim 10^{19} \mathrm{~W} / \mathrm{cm}^{2}$ over this distance, diffraction from the beam waste at focus must be limited. Self-guiding, where the laser-generated plasma causes refraction back toward the laser axis along the beam path, has been demonstrated previously in LWFA plasma using pure helium gas. We demonstrate self-guiding measurements in $\mathrm{He} / \mathrm{Ne}$ and $\mathrm{He} / \mathrm{Kr}$ gas mixtures showing self-guiding in neon and in low concentrations of krypton.
\end{abstract}




\section{INTRODUCTION}

\section{Laser wakefield acceleration}

Laser wakefield acceleration (LWFA) uses a short pulse laser to create an accelerating structure for electrons. Unlike traditional particle accelerators (which are many kilometers in length), LWFA accelerates electrons to $\mathrm{GeV}$ energies over lengths on the order of centimeters. Monoenergetic energies of up to $0.5 \mathrm{GeV}^{1}$ have been reached, and maximum energies of $2.4 \mathrm{GeV}^{*}$ have been obtained with a broad energy spread. A "table-top" accelerator using this technology would allow for the proliferation of particle accelerators in many fields. ${ }^{2}$

In order to generate LWFA, a powerful short-pulse laser is focused on a gas-filled target. As the laser pulse propagates through the gas, the gas is ionized via tunnel ionization, a quantum mechanical effect. As the laser's oscillating electromagnetic field interacts with the gas atoms, the potential wells of the bound electrons are distorted, becoming both narrower and shorter. This distortion increases the probability of ionization, allowing for the electrons to escape. ${ }^{3}$

After ionization, the laser's ponderomotive force moves the electrons away from the center of the beam (where the electric field is strongest), while the ions remain there, creating an electrical potential across the gap. This gap is called the "bubble," and the multiple bubbles left behind the laser pulse collectively are called the "wake," leading to the term "wakefield." Any electron injected trapped in the bubble would be accelerated by the potential difference. ${ }^{3}$ Figure 1 illustrates the injection of nitrogen electrons into a laser wake.

\footnotetext{
${ }^{*}$ X. Wang et al., in unpublished Proceedings of 2012 Advanced Accelerator Concepts Workshop, June 10-15, Austin, TX
} 


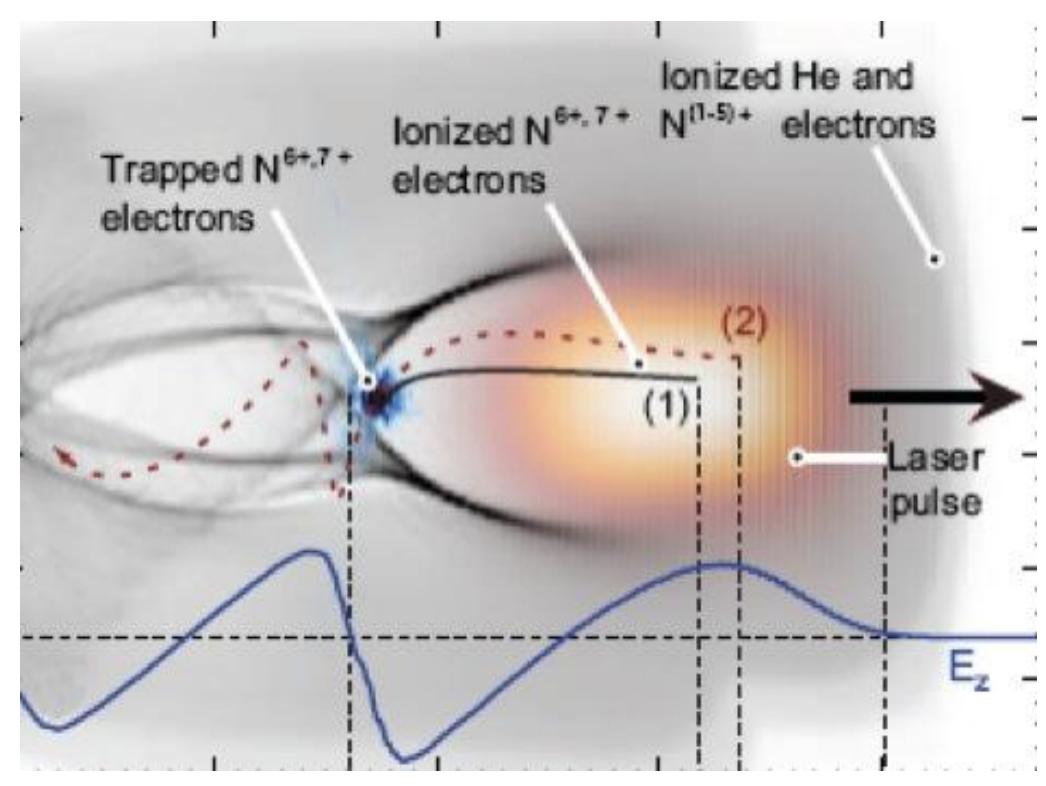

Figure 1: An illustration of electron injection during LWFA. ${ }^{3}$

\section{Self-guiding}

As a focused laser propagates through a vacuum, the light diffracts. In order to achieve LWFA, the laser beam must be guided through the plasma with minimal diffraction to maintain sufficient intensity to continue accelerating the electrons. This "self-guiding" ${ }^{4}$ can be achieved in a number of different ways, including creating a suitable index of refraction profile within the plasma itself. Light refracts towards areas of higher index of refraction, so if conditions can be created where the area outside the beam path has a lower index of refraction than the axis, the light would be refracted back towards the desired axis. ${ }^{4}$

Helium has been shown to achieve the appropriate index of refraction, $\eta$, to prevent light from diffracting from the beam path of the laser. ${ }^{5}$ Equation 1 shows the factors impacting the index of refraction of the plasma. ${ }^{6}$

$$
\eta=\left(1-\frac{1}{2} \frac{\omega_{p}^{2}}{\omega_{o}{ }^{2}}\left(1+\frac{\delta n_{e}}{n_{e}}-\frac{\left\langle a_{o}{ }^{2}\right\rangle}{2}\right)\right),
$$

where $\omega_{o}$ is the frequency of the laser, $\omega_{p}$ is the plasma electron frequency, $n_{e}$ is the electron density, and $a_{o}$ is the vector potential of the laser. ${ }^{6}$ Helium is able to achieve the desired index of refraction gradient because both He electrons can be ionized by fields achieved at the beginning of the laser pulse. Because of this, the electrons are immediately blown out into the wake, leaving only ions in the center of the bubble. ${ }^{1}$ The density is therefore high on the edges of the wake and low in the center; the $\delta n_{\mathrm{e}} / \mathrm{n}_{\mathrm{e}}$ term thus encourages a favorable index of refraction curve. In addition, the vector potential $a_{o}$ of the laser is a Gaussian; this term also leads to a favorable profile. 


\section{Experiment}

Other gases could have other effects, however. If the ionization of electrons occurs later in the laser pulse (for those electrons with higher ionization energies), the electrons will be injected continuously inside the wake. This can lead to a flat or otherwise distorted density profile, changing the effect of the $\delta \mathrm{n}_{\mathrm{e}} / \mathrm{n}_{\mathrm{e}}$ profile. This experiment will explore different concentrations of high-Z gases ( $\mathrm{Ne}$ and $\mathrm{Kr}$ ) in helium to find if self-guiding is possible in gases with more ionization states.

\section{MATERIALS AND METHODS}

\section{Laser}

This experiment took place at the Jupiter Laser Facility (JLF) at Lawrence Livermore National Laboratory (LLNL), with the Callisto laser. Callisto is a Ti:sapphire laser capable of $200 \mathrm{TW}$ of power in $60 \mathrm{fs}$. An f/8 off-axis parabolic mirror is used to focus the beam onto the target, with a spot size of $w_{0}=15 \mu \mathrm{m}$ (beam diameter $=30 \mu \mathrm{m}$ ) at the $1 / \mathrm{e}^{2}$ intensity point.

\section{Imaging system}

In order to test for self-guiding, the beam is imaged as it exits the gas cell. The light transmitted through the gas cell target during the pulse is imaged onto a 14-bit CCD camera with a 10x focusing objective. A two-lens system is set up on the axis of the beam to capture the image at the exit of the gas cell. The imaging system used is shown below in Figure 2.

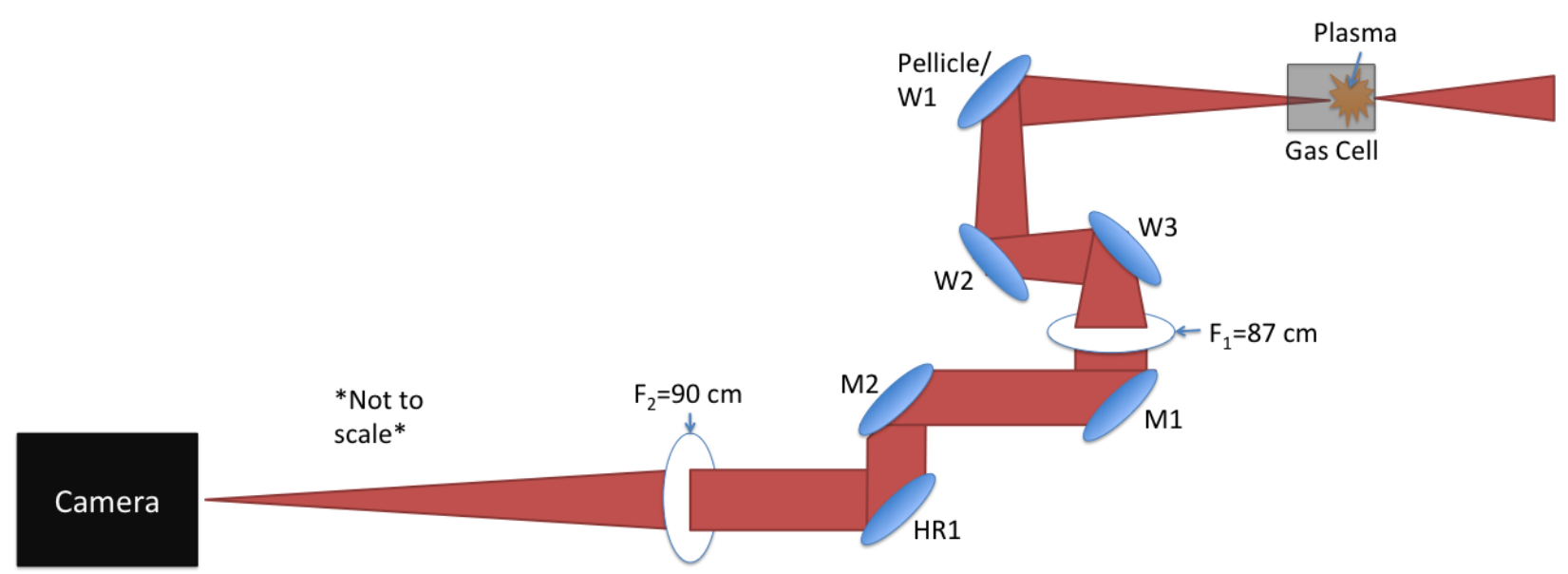

Figure 2: The imaging system which captures the transmitted beam light. The $\mathrm{W}$ optics are wedged, the $\mathrm{M}$ optics are silver mirrors, and the HR optic is highly-reflective for $775-875 \mathrm{~nm}$. The F values represent the focal lengths of the 2 lenses. The camera has a 10x microscope objective.

Originally, a pellicle was used to deflect the beam from the electron acceleration axis, but a wedge was employed later to achieve higher resolution. 


\section{Data analysis}

The images obtained during experimentation are analyzed using the MATLAB codes in Appendices A, B, and $\mathrm{C}$.

The images are delineated in the files in pixels, so the imaging system is to be analyzed for magnification and resolution. Images of the 1951 United States Air Force resolution test chart (USAF1951, Figure 3 below) are taken and analyzed using ImageJ software in order to determine both the resolution and magnification.

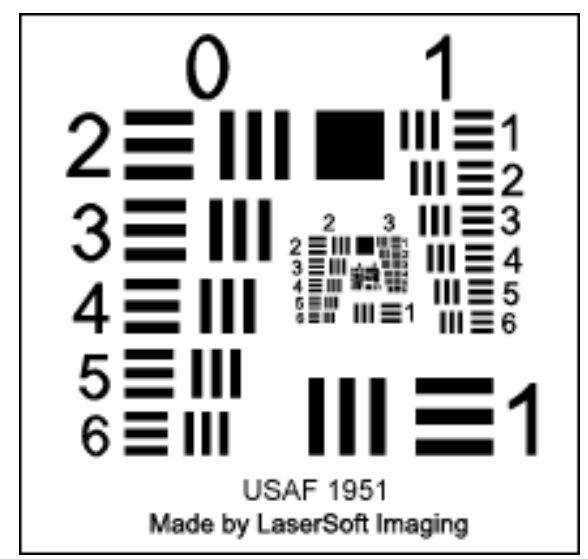

Figure 3: 1951 United States Air Force resolution test chart ${ }^{7}$

Due to a setting in the image capture software, $2^{15}$ is subtracted from each pixel in the data.

Additionally, an average of the background both horizontally and vertically is taken and subtracted from the data to attempt to eliminate background noise. In order to account for saturated pixels in some images, the MATLAB code includes a loop to test for singular saturated points and exclude them for determining local maxima.

MATLAB is used to calculate the maximum intensity and its location on the image. In the case of saturated maxima, the code finds the approximate center of the saturated region and treats that as the maximum. From there, the code takes a lineout of the row and column where the maximum occurs. Then the code finds the full width at half maximum (FWHM) by finding the location of the points within a given variation of the half maximum, and finding the difference between the leftmost and rightmost location values.

Several problems exist with this means of calculating the FWHM. First, several of the images contain saturated data (as a result of improper filtering). Though the code calculates the FWHM, the value is inaccurate as it uses a point mid-curve as the maximum intensity value, while the actual value may be much higher. In order to correct for this error, data with saturated maxima are exported into Microsoft Excel, where a Gaussian fit is performed in order to find a more accurate FWHM value. Second, some 
data shows multiple peaks. The cause of these peaks will be discussed in the Results and Conclusions sections; however, these data are manually analyzed for FWHM values based on the lineout plots.

In addition to the saturation inaccuracy, maximum intensity values are found to be less useful for other reasons. Neutral density (ND) filters and red glass (RG) filters are used in different amounts in different shots; this occurs because some experimentation was necessary before finding the correct amount of filtering for the images. The RG 830 filter used has attenuation values which are heavily dependent on wavelength, especially around $800 \mathrm{~nm}$ light. Since spectrometry data is only available for some shots, an accurate attenuation is impossible to determine. Additionally, ND filters are not entirely neutral, so the wavelength of light does affect their attenuation value, making any intensity calculations based on these values inaccurate unless the spectrum of the beam and the optical transfer function at the corresponding wavelength are measured. As a result, comparison of maximum peak values between shots with different filtering is difficult.

Spot sizes are compared to the unguided beam, which has a diameter of approximately $500 \mu \mathrm{m}$ (FWHM $\approx 300 \mu \mathrm{m})$. Figure 4 shows this image, which was obtained by allowing the $800 \mathrm{~nm}$ laser to pass through the gas cell under vacuum.

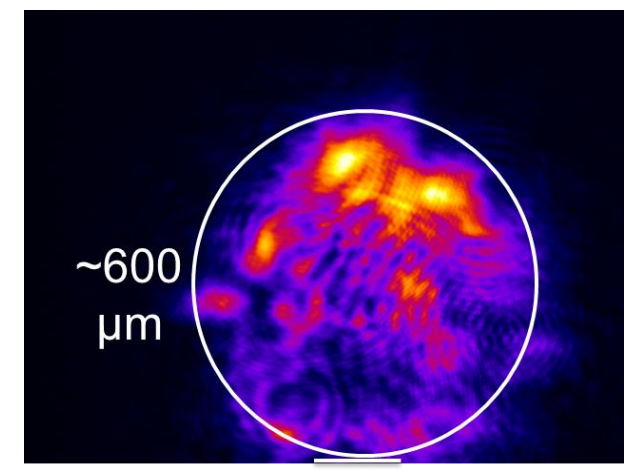

Figure 4: An image of the unguided laser beam

\section{RESULTS}

Figure 5 shows an example of an image and the subsequent MATLAB generated lineouts for neon data. 

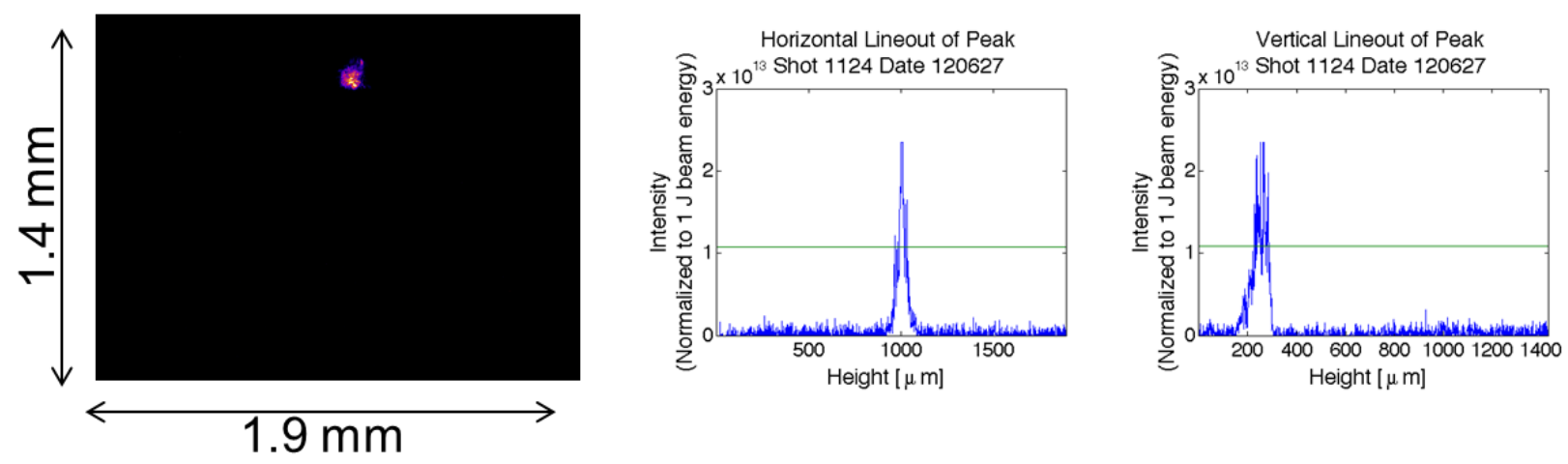

Figure 5: a) Original image of the beam. b) Horizontal lineout. c) Vertical lineout. This shot used $9.5 \%$ $\mathrm{Ne}$ in $\mathrm{He}$, with a $6.3 \mathrm{~J}$ laser pulse.

Figure 6 below shows the results of the FWHM measurements for various concentrations of $\mathrm{Ne}$ in $\mathrm{He}$ (from $9.5 \%$ to $100 \% \mathrm{Ne}$, by partial pressure).

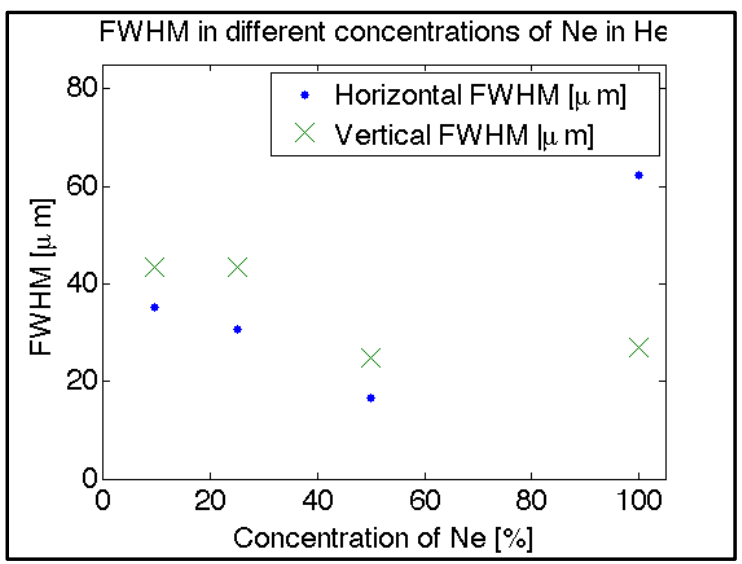

Figure 6: The beam size for various concentrations of $\mathrm{Ne}$ in He based on FWHM measurements.

Figures 7 and 8 below show typical krypton data. As Figure 7a shows, the beam has multiple bright spots, all of which have intensity on the same order of magnitude. 

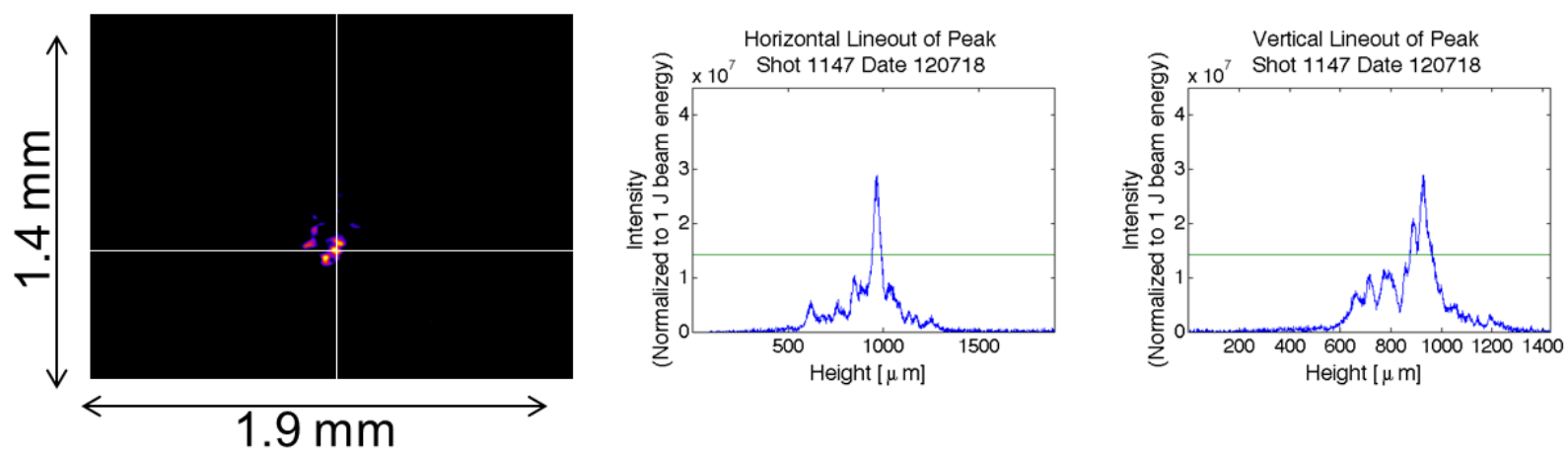

Figure 7: a) Original image of the beam. b) Horizontal lineout. c) Vertical lineout. This shot used $5 \% \mathrm{Kr}$ in $\mathrm{He}$, with a $8.5 \mathrm{~J}$ laser pulse.
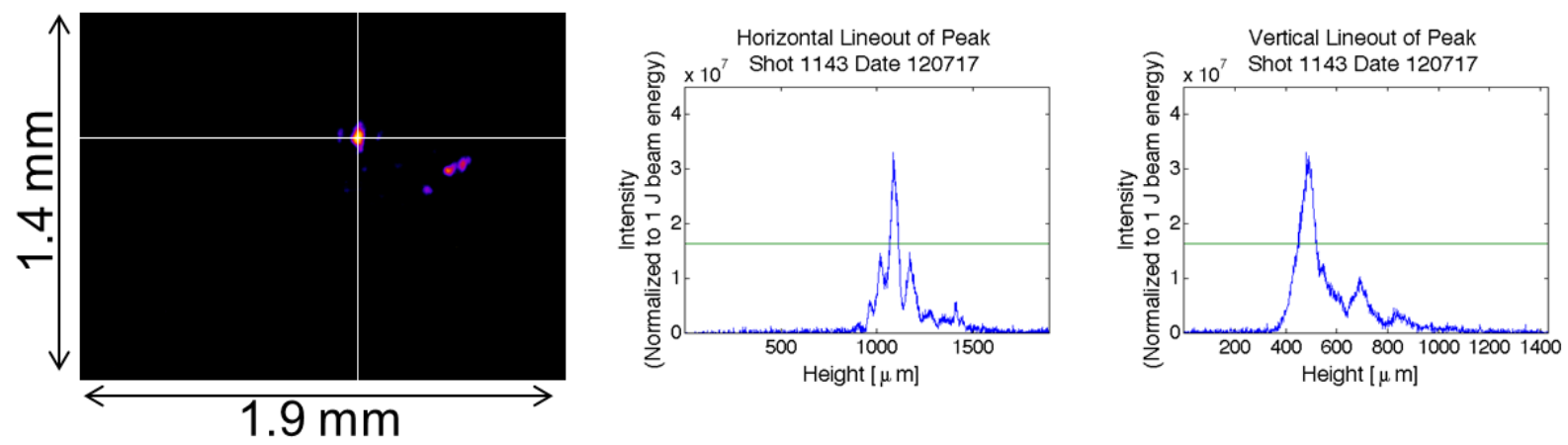

Figure 8: a) Original image of the beam. b) Horizontal lineout. c) Vertical lineout. This shot used $25 \% \mathrm{Kr}$ in $\mathrm{He}$, with a $6.2 \mathrm{~J}$ laser pulse.

\section{CONCLUSIONS}

Based on Figures 4 and 6, all neon shots are self-guided. All FWHM values are significantly less than the unguided value.

The krypton shots all show multiple peaks. There are several possible causes for these multiple peaks. As discussed in the Introduction, the self-guiding ability of the plasma is determined by Equation 1. Krypton has electrons whose ionization occurs at much higher energies; as a result, electrons are injected into the plasma closer to the center of the laser pulse. These electrons are born into the center of the bubble, which distorts the density profile. This can create a density profile which is flat across the laser spot (no self-guiding); it can also create an density profile which has multiple peaks (the edges and the center), allowing for self-guiding conditions in two different channels, causing multiple peaks. 
Another possible cause is an irregular beam profile. An observation of the beam profile (taken after the experiments were completed) shows large distortions in the beam, including a "hole." These aberrations could lead to two hot spots guiding through the plasma separately.

It is believed that krypton shows guiding up to $25 \%$ concentration. However, given the uncertainty of the data, further study into self-guiding with krypton is necessary.

\section{ACKNOWLEDGEMENTS}

There were many people who greatly influenced this experiment. First and foremost, I would like to thank Brad Pollock and Felicie Albert, the team leaders for this campaign (both at LLNL). They were invaluable as mentors, teachers, and co-researchers. Thanks also to Art Pak (LLNL) and Ken Marsh (UCLA) for all their laboratory help and ideas for experimental improvements. Steven Maricle (JLF, LLNL) was incredibly accommodating and helpful as the operator of the Callisto laser. The other interns with whom I worked (Sarah Mills, Brian Severson, and Alex Rigby) were indispensable in the lab, in addition to making the whole experience a more pleasurable one. Jessie Shaw (UCLA) gave excellent guidance during her time at the laboratory. Finally, thanks to my mentor, Siegfried Glenzer, for facilitating the experiment and for taking his time to ensure that the interns all had a rewarding experience. 


\section{APPENDICES}

\section{Appendix A: Reading .tif images, MATLAB file}

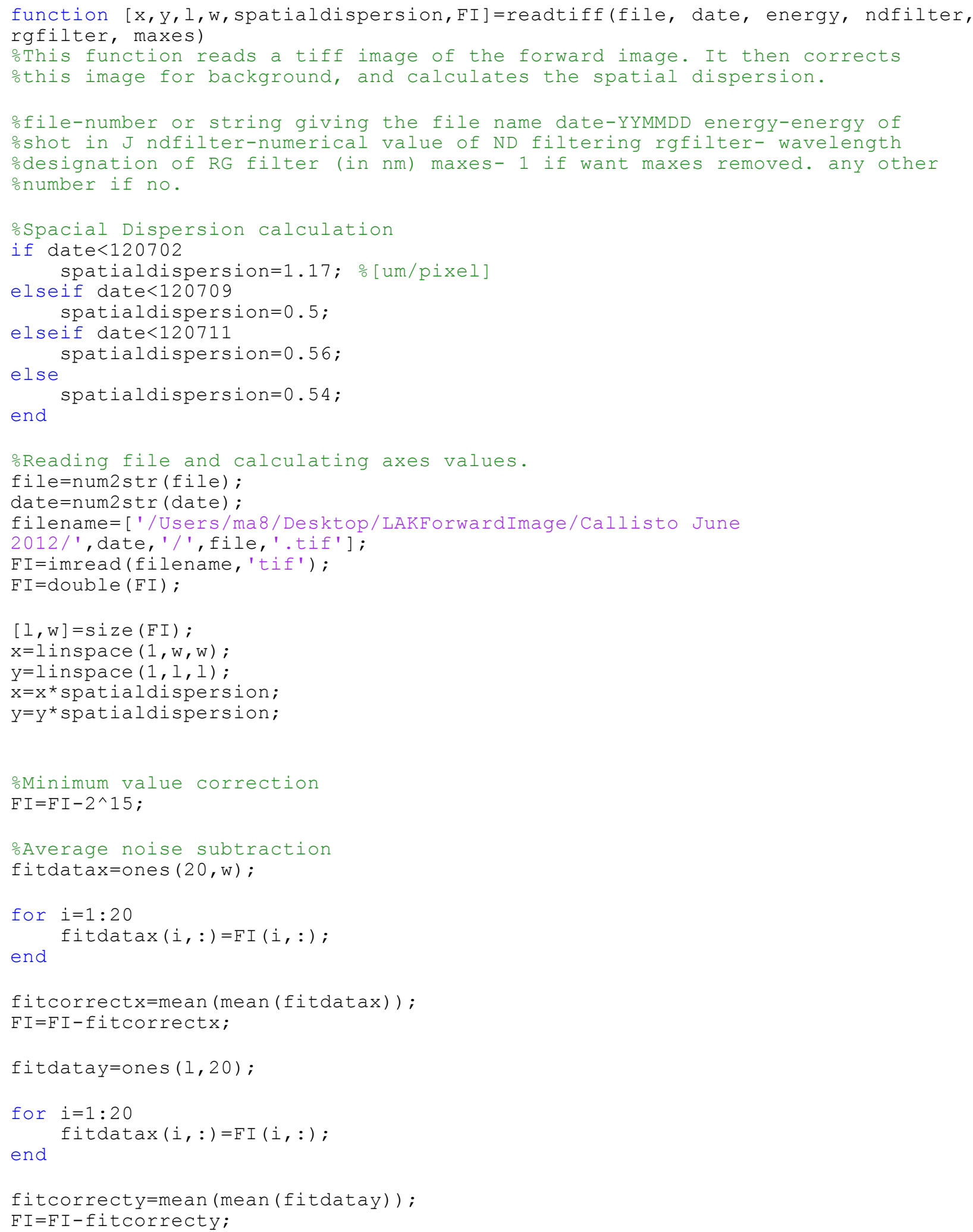




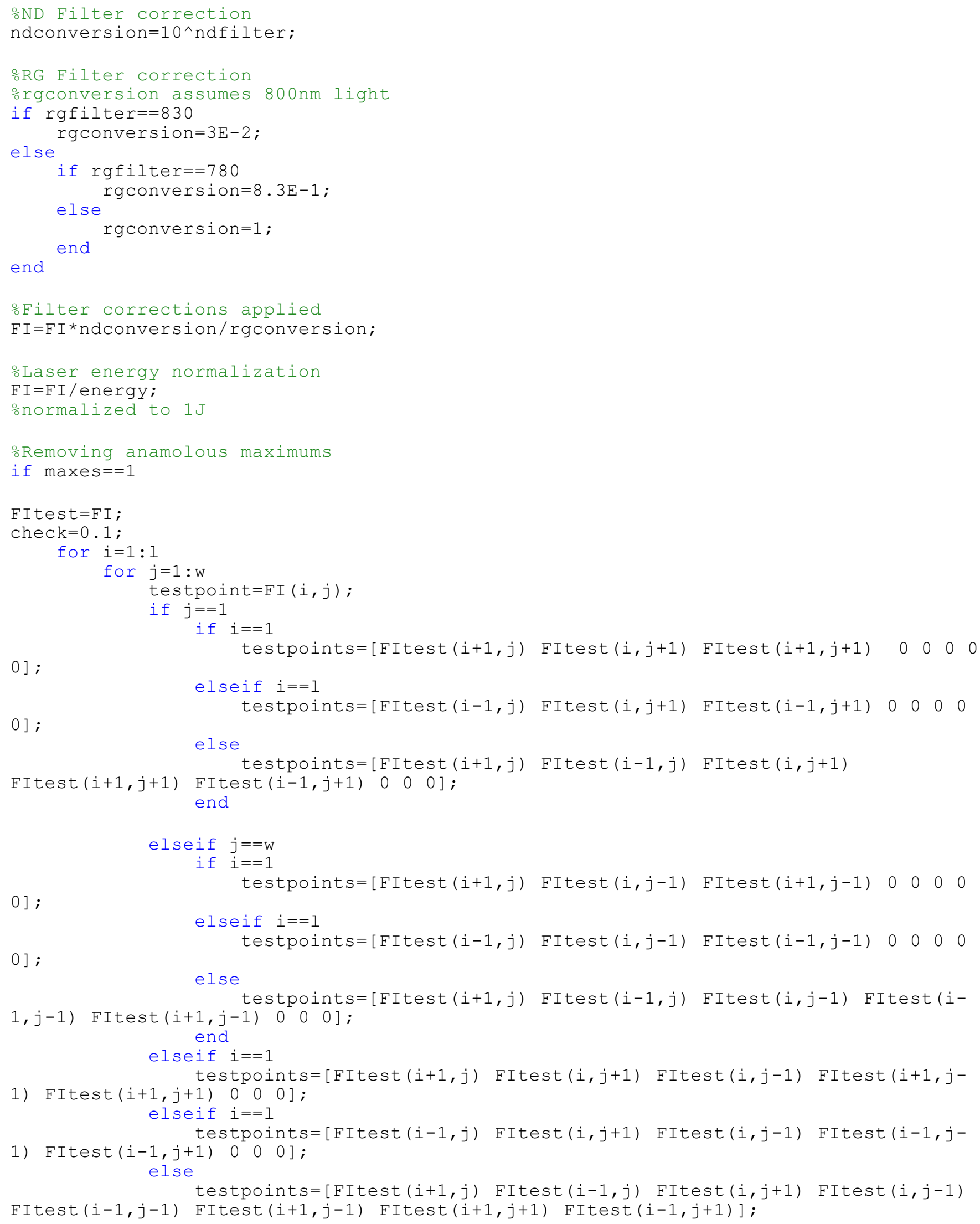




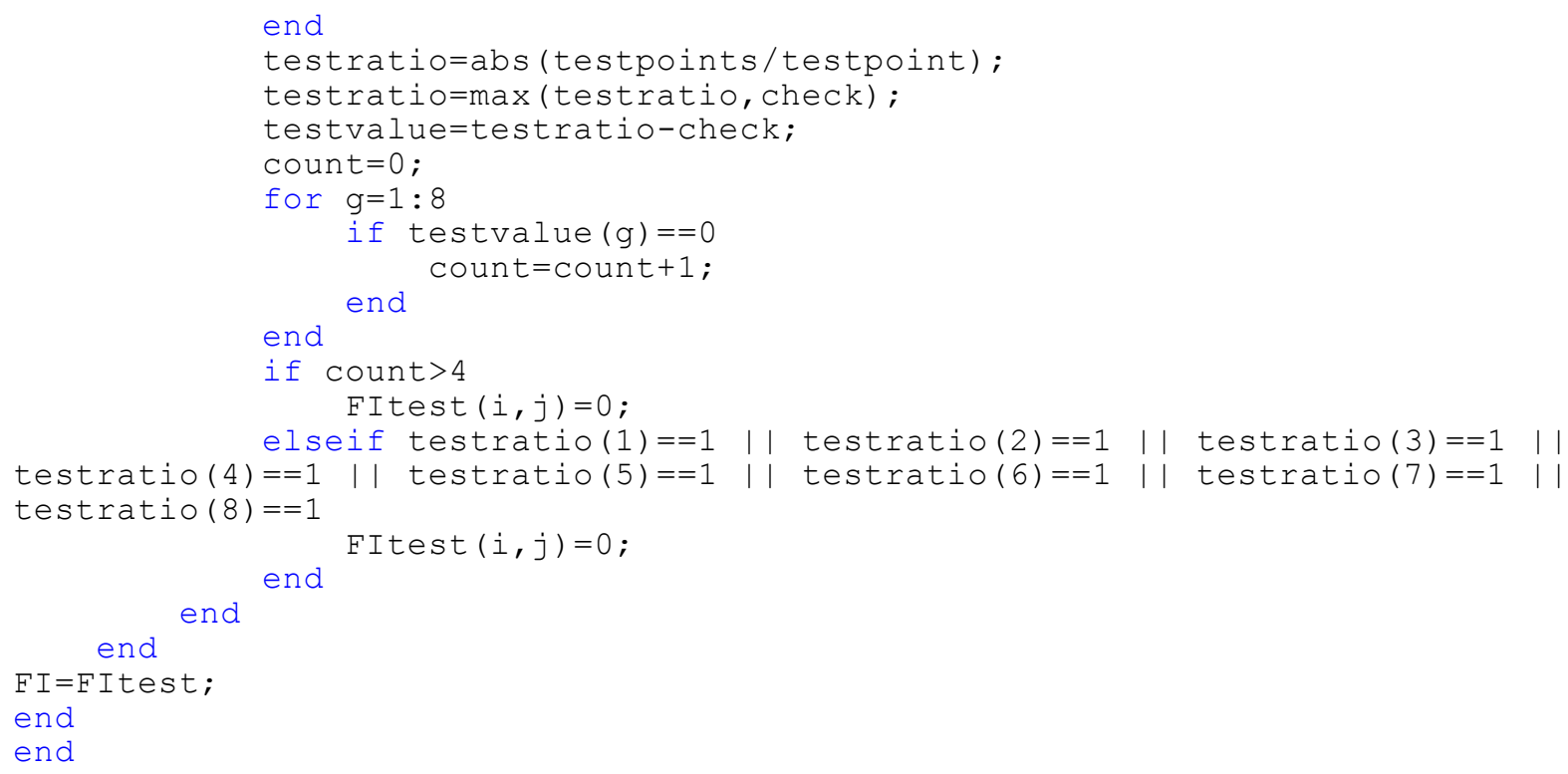

\section{Appendix B: 3D Plotting of shot data, MATLAB file}

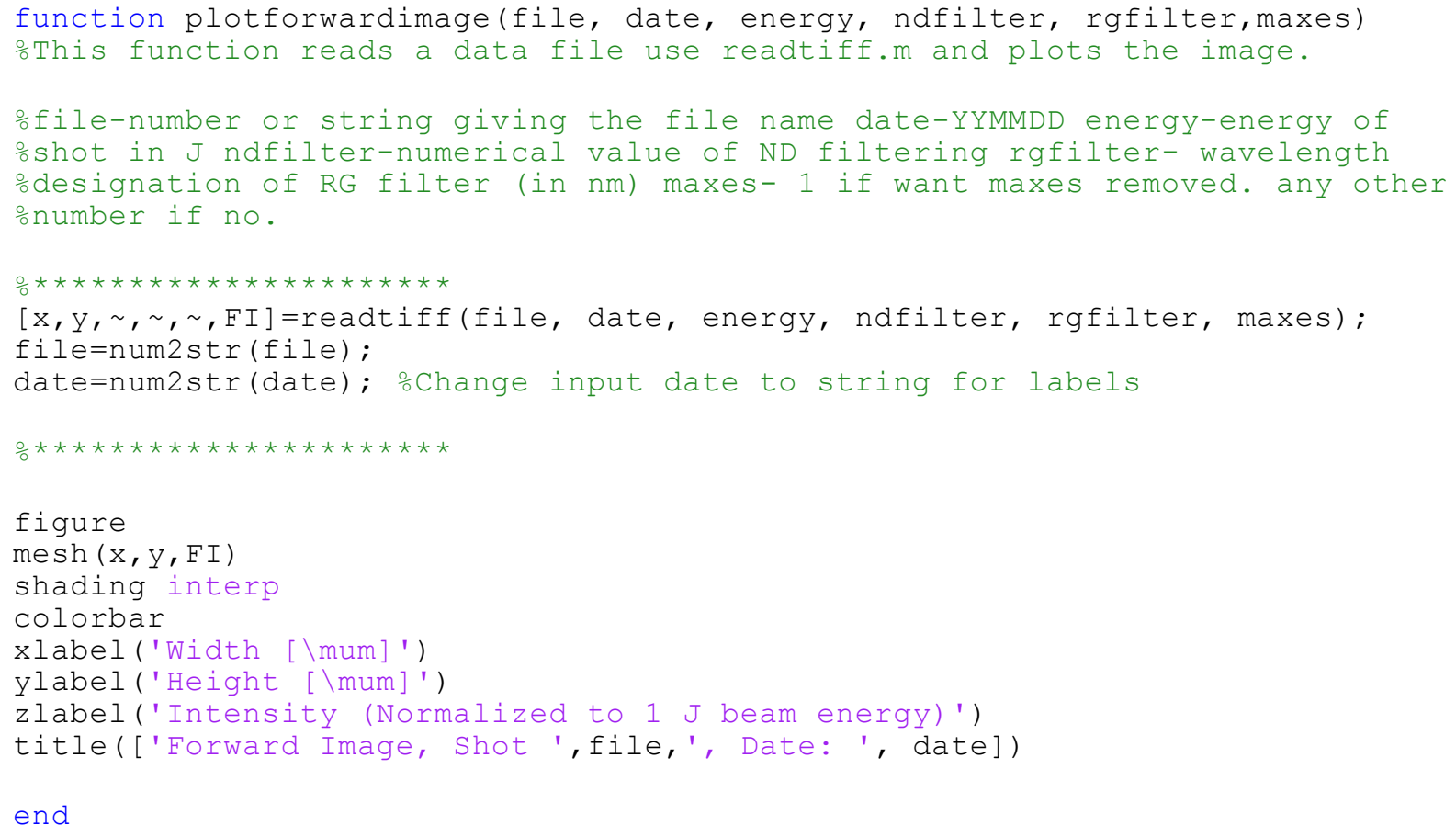

\section{Appendix C: Maximum and FWHM calculation, MATLAB file}

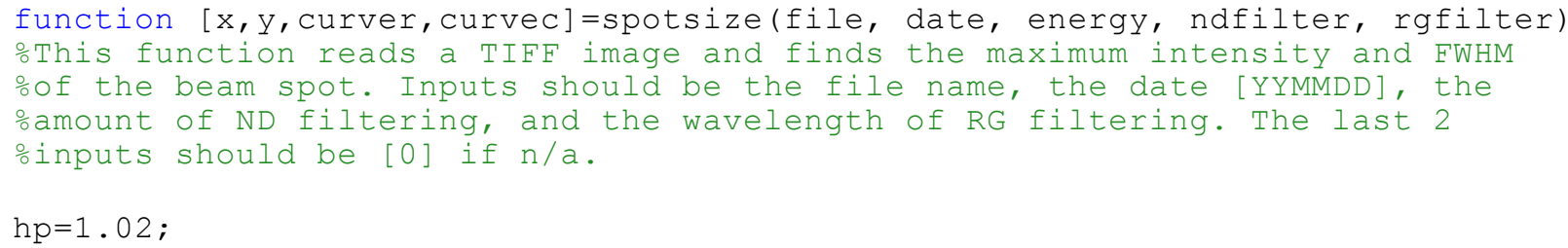




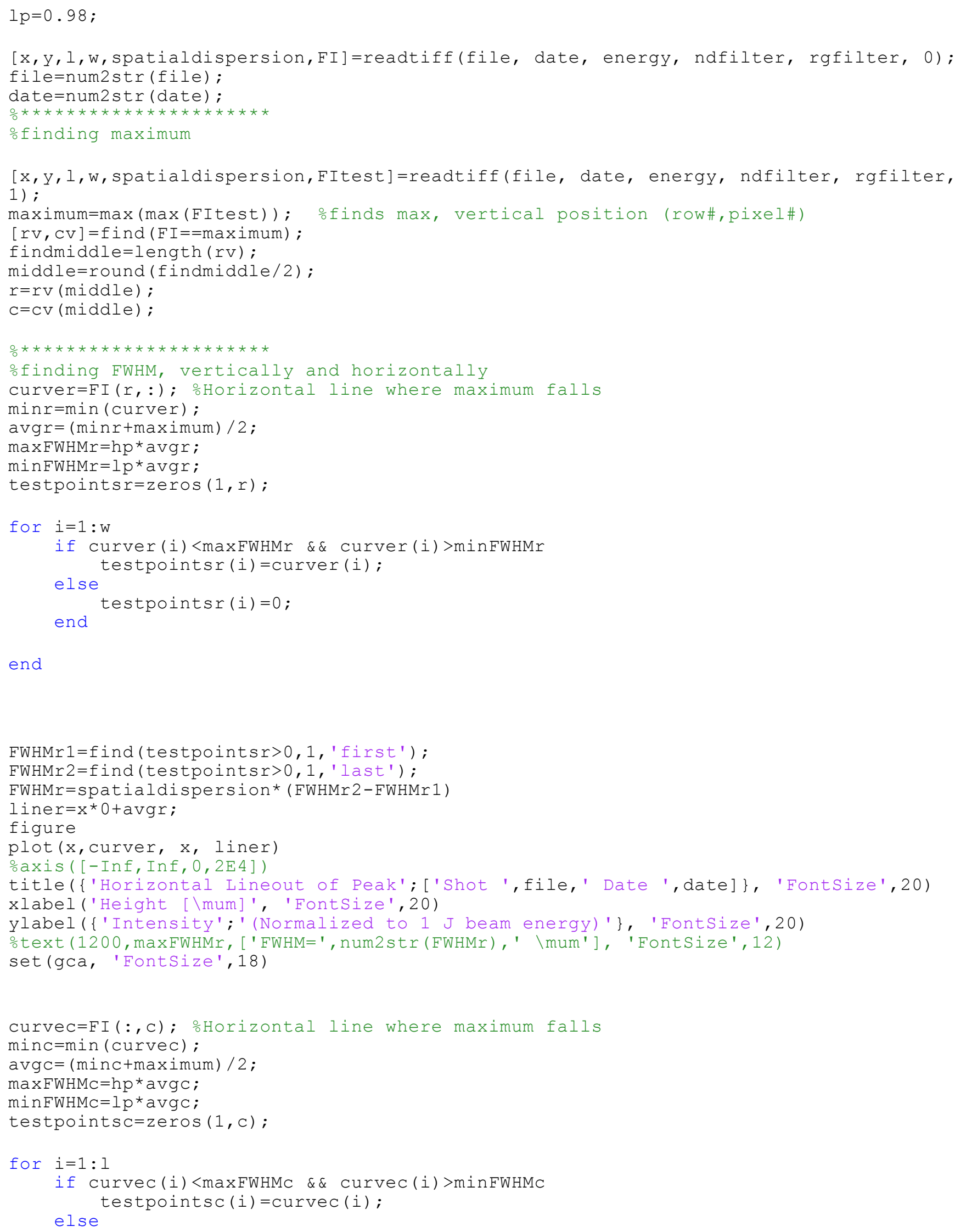




$$
\text { end } \text { testpointsc(i)=0; }
$$

end

FWHMC1=find (testpointsc>0,1, 'first');

FWHMc2=find (testpointsc $>0,1$,' last') ;

$\mathrm{FWHMC}=$ spatialdispersion * $(\mathrm{FWHMC} 2-\mathrm{FWHMC} 1)$

linec $=y^{\star} 0+\operatorname{avgc}$;

maximum

figure

$\operatorname{plot}(y$, curvec, $y, l i n e c)$

oaxis( [-Inf, Inf, 0,2E4])

title(\{'Vertical Lineout of Peak'; ['Shot ',file,' Date ', date]\}, 'FontSize',20) xlabel ('Height [\mum]', 'FontSize',20)

ylabel ( $\{$ Intensity';' (Normalized to 1 J beam energy)'\}, 'FontSize',20)

otext (1000, maxFWHMr, ['FWHM=', num2str(FWHMr),' \mum'], 'Fontsize', 12)

set(gca, 'FontSize', 18)

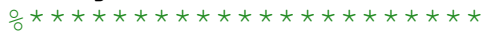

end 


\section{REFERENCES}

[1] B.B. Pollock et al. Phys. Rev. Lett. 107, 045001 (2011)

[2] C.E. Clayton et al. Phys. Rev. Lett. 105, 105003 (2010)

[3] A. Pak et al. Phys. Rev. Lett. 104, 025003 (2010)

[4] W. Leemans and E. Esarey, Phys. Today 62(3), 44 (2009); doi: 10.1063/1.3099645

[5] K.A. Marsh et al., in Proceedings of 2011 Particle Accelerator Conference, New York, NY, USA (http://www.ee.ucla.edu/ plasma/files/conference\%20proceedings/2011 Marsh pac.pdf).

[6] W.B. Mori, "The Physics of the Nonlinear Optics of Plasma at Relativistic Intensities for Short-Pulse Lasers," IEEE Journal of Quantum Electronics, vol. 33, pp.1942-1953, 1997.

[7] SilverFast, "SilverFast Resolution Target (USAF 1951) by LaserSoft Imaging" http://www.silverfast.com/show/resolution-target/en.html, 2012 